\title{
Comparison of sinew-strengthening exercise with cognitive-behavioral therapy for chronic nonspecific low back pain: protocol for a randomized controlled clinical trial
}

\section{Peng Peng}

Shuguang Hospital Affiliated to Shanghai University of Traditional Chinese Medicine https://orcid.org/0000-0001-5726-0872

Ye Zhao

Shuguang Hospital

Chengzhe Zhang

Shuguang Hospital

Zhibi Shen

Shuguang Hospital

Weian Yuan (D 444@126.com )

Shuguang Hospital

Hongsheng Zhan

Shuguang Hospital

Study protocol

Keywords: Chronic nonspecific low back pain, Cognitive-behavioral therapy, sinew-strengthening exercise

Posted Date: November 15th, 2019

DOI: https://doi.org/10.21203/rs.2.14136/v2

License: (c) (i) This work is licensed under a Creative Commons Attribution 4.0 International License. Read Full License 


\section{Abstract}

Background: Chronic low back pain is a common disease in clinic. The prevention and treatment of this disease requires a great deal of medical care resources, which is now the third medical economic burden in China. Although non-steroidal analgesics have been shown to be effective for chronic low back pain, considering the obvious side effects of these drugs, more and more patients are inclined to employ nondrug therapies. Typical examples are exercise therapy and cognitive-behavioral therapy (CBT). Exercise therapy of traditional Chinese medicine is an important part of non-drug therapy in China, such as sinewstrengthening exercise (SSE). Originating from traditional Chinese medicine exercise therapy, it has long been used for the prevention and treatment of chronic low back pain in Shuguang Hospital Affiliated to Shanghai University of Traditional Chinese Medicine (SGH), but it has not been validated by clinical trials. In this trial, we will seek to determine whether SSE is an effective and safe treatment option for persons with chronic nonspecific low back pain (CNLBP) by comparing it with CBT and explore a new method to CNLBP.

Methods/Design: In this trial, we will randomize 480 adults with CNLBP to CBT and SSE arms (240 per group). Participants in both groups will be followed for 52 weeks after randomization with home practice. Interviewers will assess outcomes 4, 8, 12, 26 and 52 weeks postrandomization. The primary measure instrument will be Roland-Morris Disability Questionnaire (RMDQ) with 26-week follow-up being the primary endpoint.

Discussion: If SSE is found to be an effective treatment option for patients with chronic back pain, it will become a meritorious addition to the current limited treatment approaches available to patients with significant psychosocial contributors to their pain.

\section{Background}

Chronic nonspecific low back pain (CNLBP) is a disease with a repeated low back pain and discomfort as its main clinical symptoms that persists for more than 3 months. Its causes cannot be attributed to pathology such as infection, tumor, osteoporosis, rheumatoid arthritis and fractures [1]. The prevention and treatment of this disease requires a great deal of medical care resources, and it is now the third medical economic burden in China [2]. CNLBP not only brings suffering to most patients, but also causes motor dysfunction and even leads to loss of self-care ability [3]. It may be related to atrophy and pathological changes of multifidus and striated muscles [4]. Both doctors and patients are dissatisfied with the status quo in the treatment of CNLBP [5] because drug adverse reactions and other factors affect the prognosis of patients [6]. Furthermore, long-term medication for chronic diseases causes huge economic pressure to many families [2]. With the change of medical model from biomedical model to biopsychosocial model, more and more attention has been paid to natural therapy. In 2017, exercise therapy is strongly recommended for treatment of chronic low back pain by the American College of Physicians (ACP). In China, traditional exercise method plays an important role in the diagnosis and 
treatment of CNLBP, and is gradually widely accepted by doctors and patients for its simplicity, practicability and curative effects.

In traditional Chinese medicine, Jin is an umbrella term of soft tissues including muscles, tendons and ligaments around the bones and joints, which is closely related to bones in physiology and pathology. It is an important rule to put equal emphasis on Jin and bones for the prevention and treatment of orthopedic diseases. Therefore, this principle should apply to all stages of Jin and bone disease. It was proposed to give priority to tendon when tendon and bone lesions occur simultaneously [7]. From clinical practice, chronic orthopedic diseases often start from the damage and pathological changes of tendon, and then involve the bones and joints. For example, patients with a disease at an early stage often have symptoms of tendons including pain, stiffness, and dysfunction, but no abnormalities were detected in bones through X-ray or CT. As the disease progresses, bone abnormalities such as hyperplasia and dislocation will occur [8]. Sinew-strengthening exercise (SSE) is a physical training method for the prevention and treatment of chronic low back pain created under the guidance of the theory of Jin, combining clinical practice with traditional Baduanjin (another common form of traditional Chinese exercise method containing eight individual movements) and modern kinesitherapy. This exercise benefits joints by physical training of tendons with simple movements as a study has shown that the thickness of multifusitis muscle changes under different positions of SSE, implying that it can effectively exercise the muscles through the coordinated movements of centrifugal and centripetal contraction [9]. It is a vital part of the comprehensive plan for prevention and treatment of CNLBP in Shuguang Hospital Affiliated to Shanghai University of Traditional Chinese Medicine (SGH). Studies have shown that this exercise can alleviate pain in the low back and leg, increase the angle of straight leg elevation and improve the functions of the low back and leg; however, its exact mechanism is not clearly clarified $[9,10]$. Cognitivebehavioral therapy (CBT), a widely-used treatment that addresses both the mind and the body, has been found to be effective for chronic low back pain including mind-body components such as relaxation training [11], emphasizing active, structured techniques to teach patients how to identify, monitor and change maladaptive thoughts, feelings and behaviors, with a focus on helping patients to acquire skills that they can apply to a variety of problems and collaboration between the patient and therapist. CBT also promotes setting and working toward behavioral goals [12].

Further research is needed on the effectiveness of SSE in CNLBP for the following reasons: (1) the large personal and societal impact of CNLBP, (2) the modest effectiveness of current treatments available, (3) the positive results of the trials in which researchers have evaluated CBT for back pain and (4) the growing popularity and safety, as well as the relatively low cost of mind-body therapies and physical exercises. To help fill this knowledge gap, we are conducting a randomized controlled clinical trial to evaluate the effectiveness and comparative effectiveness of SSE and group CBT for patients with CNLBP.

\section{Specific aims}


To evaluate the clinical efficacy of SSE in CNLBP by the measure instruments including Roland-Morris Disability Questionnaire (RMDQ) [13], back pain bothersomeness (0 to 10 scale), 36-Item Short-Form Health Survey (SF-36) and fat percentage of core muscle group.

Hypothesis: SSE will be more effective than group CBT in decreasing physical disability through RMDQ in both the short term and long term and the secondary measure instruments will be back pain bothersomeness, SF-36 and fat percentage of core muscle group. The rationale for this hypothesis is based on (1) the modest effectiveness of CBT for chronic low back pain found in past studies, (2) the positive results of the limited initial research evaluating SSE for CNLBP and (3) growing evidence that an integral part of SSE training (but not CBT training) - traditional Baduanjin - is effective for chronic low back pain [14].

\section{Methods/design}

\section{Overview}

We are conducting a randomized clinical trial in which individuals with CNLBP are randomly assigned to group SSE and CBT (Figure 1). The intervention duration of both groups lasts 12 weeks and participants will be followed for 52 weeks after randomization. The primary outcome will be assessed by RMDQ. Participants will be informed that the study researchers are comparing "two different widely used pain self-management programs that have been found helpful for reducing pain and making it easier to carry out daily activities".

[Insert here:] Figure 1 Flowchart of the trial protocol

\section{Study sample and setting}

The primary source of participants for this trial will be $\mathrm{SGH}$, a not-for-profit health-care organization with around 3.83 million outpatient visits every year in Shanghai. As needed to achieve recruitment goals, poster advertisement, telephone, social health lecturing, official website and WeChat will be employed. The subjects will be at the age of 20 to 70 years living in the areas served by SGH.

\section{Inclusion and exclusion criteria}

We are recruiting individuals from 20 to 70 years of age whose low back pain has persisted for at least 3 months. The inclusion and exclusion criteria were developed to maximize the enrollment of appropriate patients while screening out patients who have low back pain of a specific nature (for example, spinal stenosis) or a complicated nature or who would have difficulty completing the study measures or interventions (for example, psychosis). Tables 1 and 2 list the inclusion and exclusion criteria, respectively, as well as the rationale for each criterion. 


\section{Table 1 Inclusion criteria}

\section{Inclusion criteria}

To meet the diagnostic criteria for CNLBP

Age range 20 to 70 years

Low back pain duration: $\geq 3$ months

Back pain bothersomeness $\geq 4$ points ( 0 to 10 scale) with degree of low back pain measured by VAS $\geq 4$ points ( 0 to 10 scale)

Voluntary participation in research and signing the informed consent form

VAS, Visual Analogue Scale

\section{Rationales}

Study restricted to CNLBP

Chronic low back pain in children is a result of causes different from those we will study.

The elderly have a higher risk of undiagnosed serious conditions that cause low back pain.

To make sure its low back pain is chronic Severity to detect improvement

Understand the benefits and risks of this trial

\section{Table 2 Exclusion criteria}




\section{Exclusion criteria}

A disease with a clear reason that causes low back pain, i.e. lumbar intervertebral disc protrusion, discitis, ankylosing spondylitis, lumbar spinal stenosis, fracture of vertebra, vertebral tumor, vertebral tuberculosis, severe scoliosis, lumbar spondylolisthesis and osteoporosis

With other systemic pain that may affect the efficacy judgment including fibromyalgia and rheumatoid arthritis

A patient with severe underlying disease not suitable for this research, for example, severe diabetes, metabolic disease, cardiovascular, cerebrovascular, hematopoietic, digestive system, autoimmune diseases, allergic reactions and suffering from malignant tumors

Participation in research that may bring risks, spinal surgery within previous 2 years, for instance

Have obstacles to completing various questionnaires in the study: mental disorder, dementia, visual impairment, hearing disturbance, communication barrier and illiteracy

Other conditions that investigators believe not suitable for enrollment in the trial

\section{Rationales}

Back pain due to or possibly result of specific disease or condition

Condition or circumstance might confound treatment effects or interpretation of data Condition might increase the risks of adverse events

Back problem is complicated by medical or medicolegal issues

Condition might make it difficult to finish what is defined in the protocol

Investigator's comprehensive consideration

In addition, we require that participants be willing and able to attend the CBT or SSE group during the whole intervention period if assigned to one of those treatments, and to respond to follow-up questionnaires according to the protocol.

\section{Recruitment procedures}

Based on SGH's administrative and clinical electronic case history, potentially eligible candidates with a visit in the recent 3 months (or longer than 3 months) can be identified to a provider that resulted in a diagnosis consistent with CNLBP. They will be informed of this trial in different ways including advertisement, social health lecturing, or other recruitment routes. If one is interested, a research specialist will call the potential participant to ask questions; determine eligibility; clarify risks, benefits and 
requirements of the study; and request informed consent. After the signed informed consent form has been obtained, the baseline data is collected.

To ensure that all these screened study participants remain eligible at the time that the trial begins, those who consent more than 14 days prior to the start of the intervention will be re-evaluated approximately 0 to 14 days prior to the first visit to reconfirm their eligibility. The primary concern is to exclude persons who no longer have at least moderate baseline ratings of pain bothersomeness and pain-related interference with activities. Those individuals who remain suitable to this trial and give their final informed consent will be included.

\section{Randomization}

All the volunteers will be trained with procedures and notes of both SSE \& CBT by qualified investigators or instructors, and they will also be assessed whether they master these skills. After completing the baseline assessment, participants will be randomized in equal proportions to the SSE or CBT group. Each person will get a random number from 1 to 480 scheduled according to inclusion sequence. The group sequence is generated from a study biostatistician. The subjects will not be informed of their type of treatment until they are certainly included to this trial, occurring in the same site. Before a volunteer start the SSE or CBT section, the investigator will get a sealed random envelope, in which there is a card printed with the corresponding group. After one is enrolled, the investigator will open this envelope to record his/her group. Randomization will not be stratified because no confounding factors significantly influence the results. Participants will be randomized in blocks of fixed size (four) to ensure a balanced but unpredictable assignment of participants.

\section{Study treatments}

\section{Sinew-strengthening exercise}

SSE, a practical exercise method frequently used in Department of Orthopedics in SGH, is conducted as procedures in Table 3.

[Insert here:] Table 3 SSE parameters (available for download from Supplementary Files section)

\section{Cognitive-behavioral therapy}

The CBT intervention (Table 4) will consist of eight sessions in the initial 8 weeks with 2 hours each session that will provide (1) education about the role of maladaptive automatic thoughts (for example, catastrophizing) and beliefs (for example, one's ability to control pain, hurt equals harm) common in people with depression, anxiety and/or chronic pain and (2) instruction and practice in identifying and challenging negative thoughts, the use of thought-stopping techniques, the use of positive coping self- 
statements and goal-setting, relaxation techniques and coping with pain flare-ups. The intervention will also include problem-solving training that helps patients to redefine their problems and focus on daily life achievements through graded activity techniques. Participants will be given video materials of prevention and treatments of CNLBP and asked to set goals regarding their relaxation practice. During each session, participants will complete a personal action plan for activities to be completed between sessions, which will be used as logs for setting specific home practice goals and checking whether they complete during the week to be reviewed at the next week's session. In the rest 4 weeks, the investigator can receive free telephone or site consultation (outpatient) from the subjects at any time.

\section{Table 4 Content of cognitive-behavioral therapy class sessions}

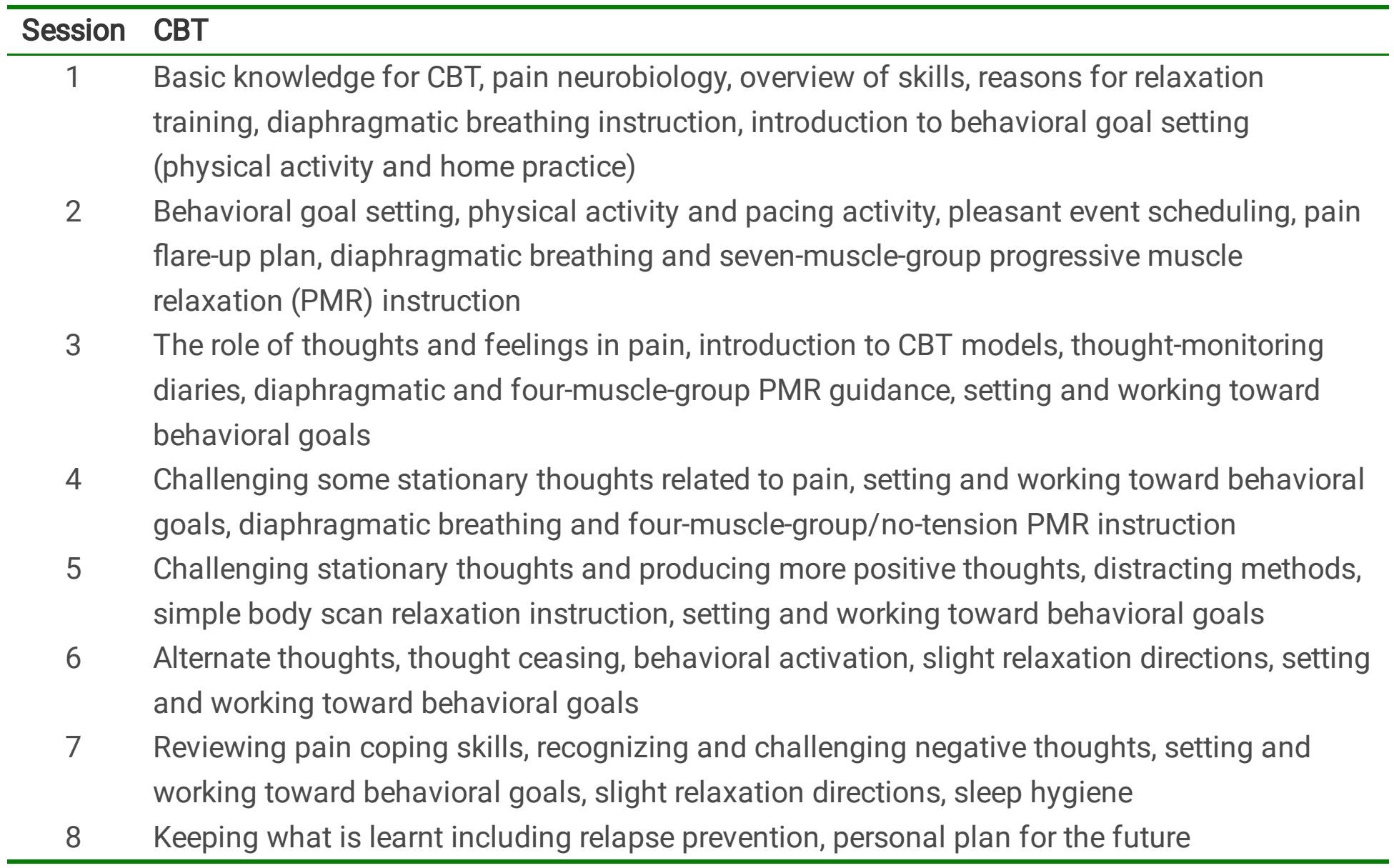

\section{Instructors}

All SSE instructors will have received either formal training in teaching SSE from a senior doctor who is experienced in treating patients for chronic low back pain in Department of Orthopedics in SGH or equivalent training. They will themselves be practitioners of SSE and will have taught SSE previously. The CBT intervention will be conducted by doctorate-level clinical psychologists with previous experience in providing $\mathrm{CBT}$ to patients with CNLBP. 


\section{Training and monitoring of instructors}

All CBT instructors will be trained in the study protocol for the CBT intervention by qualified investigators from Rehabilitation Department in $\mathrm{SGH}$, who are very experienced in administering CBT to patients with chronic pain. One of the investigators will train the SSE instructors in the adapted SSE protocol and supervise them. Each instructor will attend weekly supervision sessions, which will include discussion of positive experiences, adverse events, concerns raised by the instructor or participants and protocol fidelity. Treatment fidelity checklists highlighting the essential components for each session were created for both arms. A trained research specialist will use the fidelity checklist during live observation of every session. The research specialist will provide feedback to the supervisor to facilitate weekly supervision of the instructors.

\section{Participant retention and adherence to home practice}

Participants will receive a reminder call before each visit. They will be asked to record their daily home practice on weekly logs. Questions about their home practice during the prior week will also be included in all follow-up interviews. As SSE and CBT are different kinds of exercises, the investigator knows the group of volunteers only, but the evaluator who is not a member in the research team is kept blind. The evaluator only needs to accept the training according to the content of case questionnaires without understanding the significance and grouping of this study. To maintain interviewer blinding, adherence questions will be asked after all outcome data have been recorded.

\section{Measures}

A variety of participant baseline characteristics will be assessed, including sociodemographic characteristics, low back pain history and expectations of the helpfulness of the treatments for CNLBP (Table 5).

Table 5 Baseline and follow-up measures 
Baseline characteristics

Signing a informed consent form

Randomization

Patient characteristics (age, sex, education, height, body mass, marital status)

Back pain (pain duration, pain sites, seizure frequency, interference with activities, days of pain in previous 6 months)

Expectations for back pain improvement in general and as result of SSE or CBT

Primary outcomes

RMDQ

Secondary outcomes

Back pain bothersomeness

SF-36

Fat percentage of core muscle group Safety observation

Adverse experiences from classes or home practice

New serious health problems since entering study

Concomitant therapies

$\times$

$\times$

$\times$

$\times$

$\times$

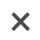

$\times$

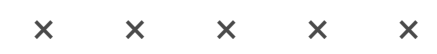

$\times \quad \times$

$\times \quad x$

$\times$

$\begin{array}{rrrrrr}\times & \times & \times & \times & \times \\ \times & \times & \times & \times & \times & \times \\ & \times & \times & \times & \times\end{array}$

Intervention-related information

Self-reported home practice

Exercise examination

Perceived helpfulness of practice

Would recommend program to friends $x \times x \times$

$x \times x \quad x$

$\times \quad \times \quad x$

$x \times x$

We will assess a core set of outcomes for patients with spinal disorders (back-related function, pain, general health status, work disability and patient satisfaction) that are consistent with the Initiative on Methods, Measurement, and Pain Assessment in Clinical Trials recommendations for clinical trials of chronic pain treatment effectiveness and safety [15]. We will measure both short-term outcomes (12 and 26 weeks) and long-term outcomes (52 weeks). Participants will be paid 20 yuan for each follow-up interview completed to maximize response rates.

\section{Primary outcome measure}

The primary outcome measure will be RMDQ. This method has been found to be reliable, valid and sensitive to clinical changes. In RMDQ, 24 questions about low back pain will be asked to the patients. 
We will analyze and report primary outcomes in two ways. First, for our primary endpoint analyses, we will compare the percentages of participants in the two groups who achieve clinically meaningful improvement ( $\geq 30 \%$ improvement from baseline) [16] at each time point (with 26-week follow-up being the primary endpoint). We will then examine, in a secondary outcome analysis, the mean differences between groups on these measures at each visit.

\section{Secondary outcome measures}

Back pain bothersomeness will be measured by asking participants to rate how bothersome their back pain has been during the previous week on a 0 to 10 scale $(0=$ "not at all bothersome" and $10=$

"extremely bothersome"). A literature showed that this bothersomeness measure is highly correlated with a 0 to 10 measure of pain intensity $(r=0.8$ to 0.9$)$ and with measures of function and other outcome measures [17]. As a result, this scale is sensitive enough in detecting changes in pain after treatment.

SF-36, known as a health survey summary, is a universal measurement scale with 8 aspects and 36 questions including physical functioning, role-physical, bodily pain, general health, validity, social functioning, role-emotional and mental health, which is one of the most commonly used standardized life quality assessment tools in the world. It has been translated to different languages since 1980s. Another health index in this short form is health transition to evaluate overall health change status in the previous year $[18,19]$.

Fat percentage of core muscle group is detected by Magnetic Resonance Hydrolipid Separation Imaging (MRHS), also named Dixon Technology, which is to perform two acquisitions by adjusting the time of echo (TE) in accordance with the difference of the hydrogen proton statnamic frequency in water molecules and fat molecules in the magnetic field. The first acquisition obtains the transverse magnetization vector of the two protons in phase, i.e. the addition of water and fat signals. The second one obtains the transverse magnetization vector with opposed phase, which is the difference image of water and fat signals. The scanning device is high field superconducting nuclear magnetic resonance, Simens skyra 3.0T MRI. The scanning parameters are as follows: horizontal axis Dixon sequence scan: TR5.36ms, TE1.24ms, flip angle: 9 degrees, FOV $500 \mathrm{~mm} \times 500 \mathrm{~mm}$, the number of layers: $44-60$ layers, layer thickness: $5.0 \mathrm{~mm}$, interval: $1.0 \mathrm{~mm}$, scanning time: $12 \mathrm{~s} \times 5$. The reason for selecting fat percentage of muscle group as a secondary outcome measure is that skeletal muscle fat infiltration is associated with the onset of spinal degenerative diseases as it leads to the substitution of contractile muscle fibers by non-contracting tissues, which may affect spine stability [20]. Degeneration of paraspinal muscles is also closely related to the recurrence of low back pain [21].

\section{Data collection, quality control and confidentiality}

Data will be collected of the questionnaires from participants by trained investigators. We will attempt to obtain outcome data from all participants in the trial, including those who never attend or drop out of the 
therapies, those who discontinue enrollment in the study and those who move away. We will collect information at every stage of recruitment, randomization and treatment so that we can report patient flow according to the CONSORT (Consolidated Standards of Reporting Trials) guidelines [22]. Data will be input to an Electronic Data Capture System (EDC) by double check. In each visit, the investigators will ask the subjects to exercise in line with what they do at home to ensure that they practice in a correct way. A patient management software (developed by Hucheng Medical Technology (Shanghai) Co., Ltd.) in the patient's cell phone is employed to improve compliance about visits, during which it reminds them of each visit when the time node is approaching. Video materials of exercises can be watched in this app and it is convenient for the subjects to follow the right movements. After home practice, the SSE participant may tick in the blank of this app every day to let the investigator know. The system will automatically send the push to the subject if it is yet not ticked at $8 \mathrm{pm}$ so as to remind exercising. For CBT subjects, some other videos are shown about fundamental knowledge of the theory, prevention, diagnosis and treatment of low back pain in this software. Although the volunteers know his/her own group, the evaluators will see the baseline and questionnaire data only in order to keep their blind status and make the results reliable. To maintain the confidentiality of patient-related information in the database, unique participant study numbers will be used to identify patient outcomes and treatment data.

\section{Protection of human participants and assessment of safety}

\section{Protection of human participants}

The SGH Ethics Committee (EC) approved this study.

\section{Safety monitoring}

This trial will be monitored for safety by an independent Data and Safety Monitoring Board (DSMB) composed of a primary care physician experienced in $\mathrm{CBT}$, a biostatistician and a clinical psychologist with experience in treating patients with chronic pain.

\section{Adverse events}

We will collect data on adverse events (AEs) from several sources: (1) reports from the CBT and SSE instructors of any participants' experiences of concern to them; (2) safety evaluation from laboratory examination; and (3) spontaneous reports from participants. The project coinvestigators and a SGH primary care internist will review AE reports from all sources weekly. Any serious adverse events (SAEs) will be reported promptly to the SGH EC, the DSMB and National Medical Products Administration (NMPA). AEs that are not serious will be recorded and included in regular DSMB reports. Any identified deaths of participants will be reported to the DSMB chair within 7 days of discovery, regardless of attribution. 


\section{Stopping rules}

The trial will be stopped only if the DSMB believes that there is an unacceptable risk of SAEs in one or more of the treatment arms. In this case, the DSMB can decide to terminate one of the arms of the trial or the entire trial.

\section{Sample size and detectable differences}

Our sample size was chosen to ensure adequate power to detect a statistically significant difference between the two groups. We specified our sample size on the basis of the expected percentage of patients with a clinically meaningful improvement measured with the primary outcome measure (RMDQ) at the 26 -week assessment (that is, at least $30 \%$ relative to baseline) [16].

A literature showed that $55 \%$ of CBT treatment group had clinically meaningful improvement on the RMDQ at 26 weeks [16]. We will have at least $80 \%$ power to detect a significant difference between SSE and CBT on the RMDQ if the ratio of SSE that improves with clinical meaning will be $20 \%$ higher than that of CBT, which means that $66 \%(55 \%+55 \% * 20 \%)$ of SSE group will have clinically meaningful improvement on the RMDQ at 26 weeks from our expectation.

The effectiveness rates of these two groups are compared. Assuming a $20 \%$ loss to follow-up, we plan to recruit a sample of 576 participants (288 per group). We will need 480 participants ( 240 in each group) to achieve $80 \%$ power to find SSE treatment different from CBT group on the RMDQ according to the formula

$$
n_{c}=n_{t}=\frac{\left(z_{1-\alpha}+z_{1-\beta}\right)^{2}\left[p_{t}\left(1-p_{t}\right)+p_{c}\left(1-p_{c}\right)\right]}{(\varepsilon-\Delta)^{2}}
$$

in which $a=0.05, \beta=0.2, p_{t}$ (the effectiveness percentage of test group) equals to $66 \%, p_{c}$ (the effectiveness percentage of control group) equals to $55 \%$, and $\varepsilon=p_{t}-p_{c}$, that is to say, the rate difference that should be detected should at least be $11 \%$ and it is assumed that the optimum value $\Delta=0$ [23].

The primary outcome will be tested at the $\mathrm{P}<0.05$ level at each time point because they address separate scientific questions.

\section{Statistical analyses}

In our comparisons of treatments based on the outcome measures, we will analyze outcomes assessed at all follow-up time points. We will conduct the following set of analyses for both the continuous outcome score and the binary outcome (clinically significant change from baseline) including all followup time points $(4,8,12,26$ and 52 weeks). The SSE treatment will be deemed successful only if the 26- 
week time point comparisons are significant. The other time points will be considered secondary evaluations.

We will use an intent-to-treat approach in all analyses; that is, the assessment of individuals will be analyzed by randomized group, regardless of participation in any classes. In this trial, if a patient does not complete the treatment, the data will be carried forward with the last observation. This analysis minimizes biases that often occur when participants who do not receive the assigned treatments are excluded from analysis.

\section{Discussion}

In this trial, we will seek to determine whether an increasingly popular approach to dealing with stressSSE-is an effective and safe treatment option for persons with CNLBP. Because it mainly focuses on physical exercise, SSE has the potential to alleviate low back pain. We will also compare the effectiveness of SSE with that of CBT, which has been found to be effective for low back pain but mainly employed in SGH. If SSE is found to be effective and safe for those who suffer from chronic low back pain, it can be generalized to medical routine in more sites.

There are a lot of traditional natural therapies including herbs, acupuncture, tuina or moxibustion in China. SSE is a noninvasive exercise therapy without gastrointestinal reactions and injuries to human body. Its training intensity is relatively low so that the patients are likely to follow the doctor's advice to persist in training, which improves efficacy.

\section{Trial Status}

The protocol version number is V1.0 and its date is 20150423. Recruitment will start in October 2019 and will be completed in June 2021 as expected.

\section{Abbreviations}

ACP: the American College of Physicians; AE: Adverse event; CBT: Cognitive-behavioral therapy; CNLBP: Chronic nonspecific low back pain; CONSORT: Consolidated Standards of Reporting Trials; DSMB: Data and Safety Monitoring Board; EC: Ethics Committee; EDC: Electronic Data Capture System; MRHS: Magnetic Resonance Hydrolipid Separation Imaging; NMPA: National Medical Products Administration; PMR: Progressive muscle relaxation; RMDQ: Roland-Morris Disability Questionnaire; SAE: Serious adverse event; SF-36: 36-Item Short-Form Health Survey; SGH: Shuguang Hospital Affiliated to Shanghai University of Traditional Chinese Medicine; SSE: sinew-strengthening exercise; TE: time of echo

\section{Declarations}

\section{Competing interests}


The authors declare that they have no competing interests.

\section{Acknowledgements}

Acknowledgment is given to staff and students at Shuguang Hospital Affiliated to Shanghai University of Traditional Chinese Medicine for their contributions to the implementation of this study.

\section{Funding}

This trial was funded by National Key New Drug Creation Project, the standardized clinical research platform construction of Chinese traditional medicine new drug for viral hepatitis (No. 2017ZX09304002) and Special Topic of Clinical Research of Shanghai Health Committee (201940063).

\section{Authors' contributions}

$\mathrm{ZS}, \mathrm{WY}$ and $\mathrm{HZ}$ designed the study. WY is the principal investigator of the study. $\mathrm{YZ}$ wrote the SSE manual. CZ wrote the CBT manual. PP is the primary author of this manuscript. WY made significant contributions to the writing. All authors read and approved the final manuscript.

\section{Ethics approval and consent to participate}

The protocol for this trial has been approved by the Independent Review Board of SGH (2015-384-1201). All participants will be required to give their informed consent before enrollment in this study.

\section{Consent for publication}

All presentations of case reports have consent to publish.

\section{Availability of data and material}

Data sharing not applicable to this article as no datasets were generated or analysed during the current study.

\section{References}

[1] Wang X, Shu S, Fang FF, Gu W: Correlation of lumbar vertebrae position perception to psoas muscles endurance and pain in chronic non-specific low back pain patients. Chin J Rehabil Theory Pract 2017, 23(7):820-823 
[2] Yang G, Wang Y, Zeng Y, Gao GF, Liang X, Zhou M, Wan X, Yu S, Jiang Y, Naghavi M, Vos T, Wang H, Lopez AD, Murray CJ. Rapid health transition in China, 1990-2010: findings from the Global Burden of Disease Study 2010. Lancet 2013, 381(9882):1987-2015

[3] Expert Group in Chinese Society of Rehabilitation Medicine Spinal Cord Committee: Expert consensus on acute/chronic nonspecific low back pain in China. Chinese Journal of Spine and Spinal Cord 2016, 26(12):1134-1138

[4] Rezazadeh F, Taheri N, Okhravi SM, Hosseini SM: The relationship between cross-sectional area of multifidus muscle and disability index in patients with chronic non-specific low back pain. Musculoskelet Sci Pract 2019, 42:1-5

[5] Mateu M, Alda O, Inda MD, Margarit C, Ajo R, Morales D, van-der Hofstadt CJ, Peiró AM: Randomized, controlled, crossover study of self-administered Jacobson relaxation in chronic, nonspecific, low-back pain. Altern Ther Health Med 2018, 24(6):22-30

[6] Mohammed AA; Sally D; Mohammed Q; Abu S; Aksh C: Effects of McKenzie and stabilization exercises in reducing pain intensity and functional disability in individuals with nonspecific chronic low back pain: a systematic review. Journal of physical therapy science 2019, 31(7):590-597

[7] Zhou N, Fang M, Zhu QG, Shen GQ, Jiang SY, Cheng YW, Liu KP: Biomechanical evaluation of waist back muscle for Tuina manipulation treating lumbar intervertebral disc protrusion. China Journal of Traditional Chinese and Pharmacy 2012, 27(3):562-566

[8] Yuan WA囚Zhan HS囚Du GQ: Clinical significance of concept of"tendon domination and bone subordination"in diagnosis and treatment of chronic tendon and bone diseases. SH. J.TCM2019, 53(9):12-15

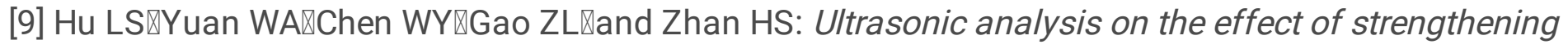
exercise with strong tendon on lumbosacral multifidus muscles. China J Orthop Trauma 2017, 30(11):1034-1038

[10] Zhang JQ, Wang XD, Meng XY, Zhang YL: Research on effects of "Qiangjingushengong" rehabilitation exercise in patients with lumbar disc herniation in remission stage. China Journal of Nursing Administration 2013, 13(10):736-737

[11] Williams AC, Eccleston C, Morley S: Psychological therapies for the management of chronic pain (excluding headache) in adults. Cochrane Database Syst Rev 2012, 11:CD007407

[12] Aggarwal VR, Lovell K, Peters S, Javidi H, Joughin A, Goldthorpe J: Psychosocial interventions for the management of chronic orofacial pain. Cochrane Database Syst Rev 2011, 11:CD008456.

[13] He G, Zhang JX, Shen CL, Yang QG: Reliability of Chinese version of the Rowland-Morris disability questionnaire for evaluating patients with low back pain. Chinese Journal of Spine and Spinal Cord 2005, 
[14] Liu WH, Shi XM, Yu J, Wang XJ, Li GX, Xue YY, Zhang L, Li M: Exercise therapy in prevention and treatment of navy personnel with chronic low back pain. Med \& Pharm J Chin PLA 2018, 30(11):10-13

[15] Dworkin RH, Turk DC, Farrar JT, Haythornthwaite JA, Jensen MP, Katz NP, Kerns RD, Stucki G, Allen RR, Bellamy N, Carr DB, Chandler J, Cowan P, Dionne R, Galer BS, Hertz S, Jadad AR, Kramer LD, Manning DC, Martin S, McCormick CG, McDermott MP, McGrath P, Quessy S, Rappaport BA, Robbins W, Robinson JP, Rothman M, Royal MA, Simon L, Stauffer JW, Stein W, Tollett J, Wernicke J, Witter J: Core outcome measures for chronic pain clinical trials: IMMPACT recommendations. Pain 2005, 113.9-19

[16] Ostelo RW, Deyo RA, Stratford P, Waddell G, Croft P, Von Korff M, Bouter LM, de Vet HC: Interpreting change scores for pain and functional status in low back pain: towards international consensus regarding minimal important change. Spine (Phila Pa 1976) 2008, 33:90-94

[17] Dunn KM, Croft PR: Classification of low back pain in primary care: using "bothersomeness" to identify the most severe cases. Spine (Phila Pa 1976) 2005, 30:1887-1892

[18] Ward MM, Hu J, Guthrie LC, Alba M: Testing the construct validity of a health transition question using vignette-guided patient ratings of health. Health Qual Life Outcomes2018, 16(1):2

[19] Zhang YL, Chen ZQ, Sun Y, Zhou FF: Correlation and change of quality of life and neurological function outcome for cervical spondylotic myelopathy after surgery. Chinese Journal of Spine and Spinal Cord 2016, 26(9):782-790

[20] Hamrick MW, McGee-Lawrence ME, Frechette DM: Fatty infiltration of skeletal muscle: mechanisms and comparisons with bone marrow adiposity. Front Endocrinol (Lausanne) 2016, 7:69

[21] D'hooge R, Cagnie B, Crombez G, Vanderstraeten G, Dolphens M, Danneels L: Increased intramuscular fatty infiltration without differences in lumbar muscle cross-sectional area during remission of unilateral recurrent low back pain. Man Ther 2012, 17(6):584-588

[22] Boutron I, Moher D, Altman DG, Schulz KF, Ravaud P, CONSORT Group: Extending the CONSORT statement to randomized trials of nonpharmacologic treatment: explanation and elaboration. Ann Intern Med 2008, 148:295-309

[23] Jin PH, Chen F: Medical Statistics Method. 3rd edition. Shanghai: Fudan University Press; 2009

\section{Figures}




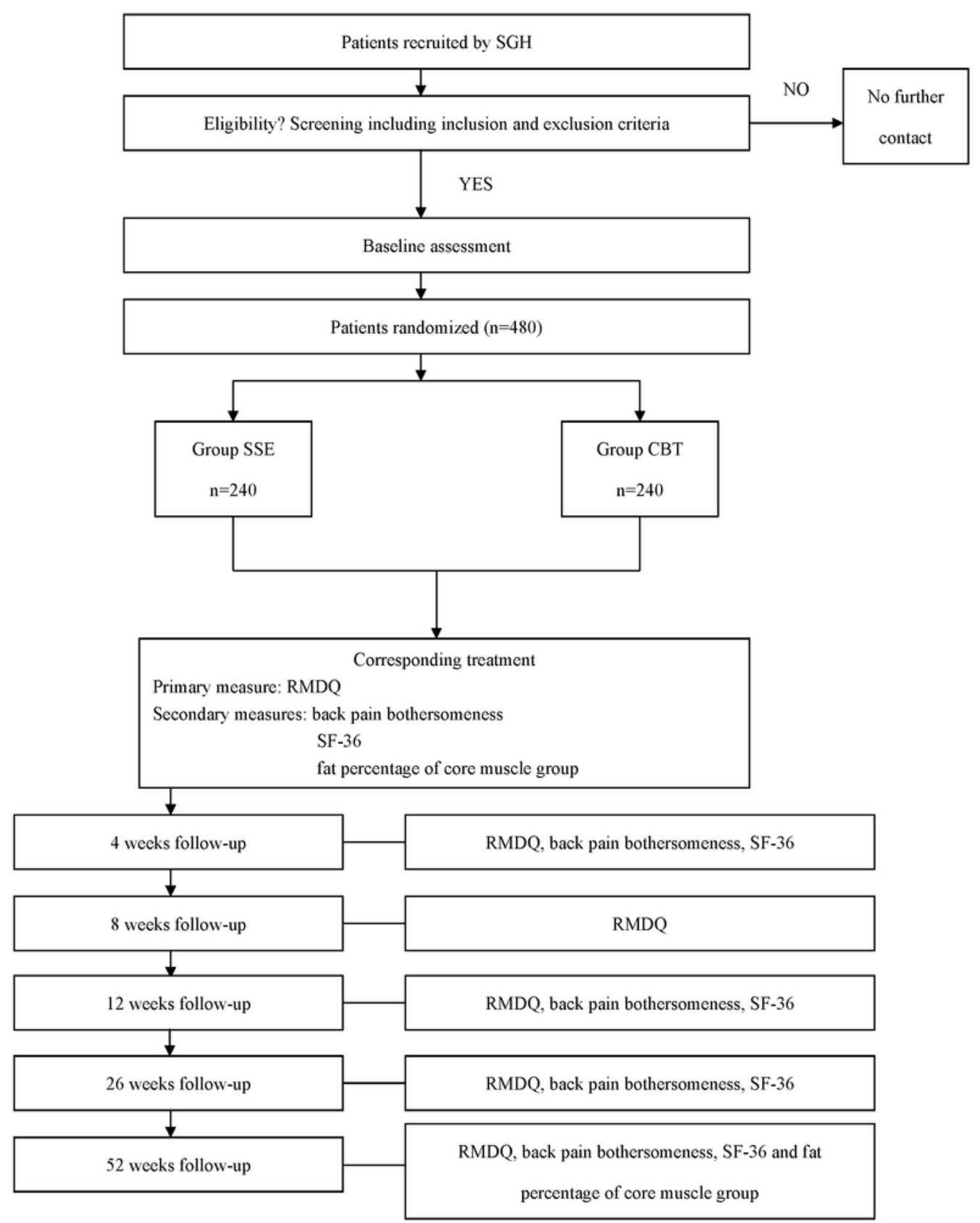

\section{Figure 1}

Flowchart of the trial protocol

\section{Supplementary Files}

This is a list of supplementary files associated with this preprint. Click to download. 
- checklist.doc

- Table3.docx

Page 19/19 